practice was created as a consensus document to establish a unique and consistent identification system for animal-drawn vehicles on public roads. Many outreach efforts engage the Anabaptist communities to adopt these national practices with varying degrees of compliance depending upon the geographic region and local culture.

Methods The process relied on a social marketing framework. Social marketing techniques are used to influence a target audience to voluntarily accept, reject or modify a behaviour for the benefit of individuals, groups, or society as a whole. The study was directed by safety staff at The Ohio State University and utilised a five-step approach including: a needs assessment, identification of potential lighting and marking schemes, consensus among the various stakeholders, revision of an existing engineering practice, and dissemination of the standard to encourage adoption of a uniform lighting and marking pattern.

Results Nearly two years was needed to develop consensus among Anabaptist stakeholders and members of the professional engineering society. The lighting scheme incorporated elements from each group, and was evaluated for its practical application, affordability, and visibilty.

Conclusions The revised Engineering Practice enhanced the previous national standard for animal-drawn vehicles and created a consistent lighting and marking pattern. Building consensus between the two groups proved to be a challenging task, yet their collective efforts identified plausible solutions for a roadway safety issue.

\section{HEALTH SECTOR'S ROLE IN ADDRESSING GAPS IN THE PREVENTION OF TRAFFIC INJURIES}

${ }^{1}$ Mitchell I Wolfe, 'Maeve McKean, 'Gabrielle Lamourelle, 'Colin Mclff, ${ }^{2}$ David Sleet, ${ }^{3}$ Deana Jordan-Sullivan. ${ }^{1}$ Department of Health and Human Services (HHS), Office of Global Affairs, USA; ${ }^{2}$ Centres for Disease Control and Prevention, USA; ${ }^{3}$ Embassy, Geneva, USA

\subsection{6/injuryprev-2016-042156.892}

Background Despite substantial progress in some countries in traffic injuries prevention enormous gaps remain which the health sector can play a critical role addressing.

Description of the problem Road traffic injuries claim the lives of over 1.2 million people and seriously injure millions more each year. These largely preventable injuries lead causes of death worldwide of people ages 15-29.

Governments play a critical role improving road safety through leading multisectoral responses. However, overcoming the current achievement plateau, with injury numbers rising mainly in low- and middle-income countries, requires the health sector to increase its leadership role. Since 2004, world leaders have passed six resolutions at the UN General Assembly on Improving Road Safety, working to increase the holistic response. Although Ministers of Health last passed a resolution at the World Health Organisation on traffic injuries prevention over ten years ago, the health sector has played a critical role establishing injury data systems and the evidence base for effective interventions. The Sustainable Development Goal (SDG) target to reduce road traffic deaths and injuries by $50 \%$ by 2020 offers an opportunity for countries to work multilaterally to organise around key recommendations for actions to address current gaps. National governments would benefit from having a multisectoral strategy, including a strong health presence, to prevent and respond to traffic injuries.

Results We know what works to prevent traffic-related injuries; now is the time to implement those strategies, worldwide, for example, improving data collection and analysis for decision making; and public education on emergency response. Beyond the technical knowledge, there is also a critical role for health ministries to bring increased visibility to the public health aspects of road safety and to how health can better integrate into the multisectoral response. Countries should capitalise on the SDG targets agreement and convene various sectors-health, transportation, education, justice-to strengthen implementation of contextually-relevant best practices. The health sector can play a leading role in this process, applying its health systems and health promotion expertise.

Conclusions Road traffic injuries exert an enormous toll on national health systems. Strong health sector involvement and leadership can galvanise action on global and national health agendas. The UNGA resolutions, the Decade of Action on Road Safety Plan, and the 2015 Brasilia Declaration on Road Safety can all be leveraged to mobilise accelerated multisectoral approaches to save millions of lives.

\section{THE MAXIMUM ABBREVIATED INJURY SCALE AS A PREDICTOR OF SEVERE/FATAL INJURIES IN BELGIAN ROAD VICTIMS}

${ }^{1}$ Nina Nuyttens, ${ }^{2}$ Griet Van Belleghem, ${ }^{1}$ Emmanuelle Dupont, ${ }^{1} J u l i e n$ Leblud, ${ }^{2}$ Stefanie Devos, ${ }^{2}$ Koen Putman, ${ }^{1}$ Wouter Van den Berghe. ${ }^{1}$ Belgian Road Safety Institute, Belgium; ${ }^{2}$ Vrije Universiteit Brussel, Interuniversity Centre for Health Economics Research, Belgium

\subsection{6/injuryprev-2016-042156.893}

Background In 2013, the EC (European Commission) adopted a new definition of seriously injured road victims. All road victims with a MAIS score of 3 or more (MAIS3+) are considered as severely injured. This new definition will coexist along with the conventional definition of severely injured, namely persons who stay at least 24 hours in hospital.

Most EU countries will calculate the number of MAIS3 + victims on the basis of (national) hospital data. In Belgium, the Belgian Road Safety Institute and the Vrije Universiteit Brussel have been granted jointly access to national hospital data with detailed injury information for three consecutive years (2009-2011). Consequently, Belgium is able to express the severity of injuries in terms of MAIS, but also in terms of other severity scales such as ISS ( Injury Severity Scale), NISS (New Injury Severity Scale) and ICISS (ICD-9- Based Injury Severity Score). The purpose of this project is to make a mutual comparison of these scales.

Methods The different injury severity scales will be tested and compared as predictors of severe and fatal injuries, based on a dataset of approximately 70.000 road victims. The comparison between the different injury severity scales will be achieved both by exploratory analyses (i.e. descriptive tables and scatterplots) and by fitting generalised linear models with in-hospital mortality as dependent variable and each severity scale separately as a predictor variable. Other independent variables such as age, gender, road user type will also be investigated. The estimates, discrimination ability and calibration of the model containing the MAIS scale will be compared to models containing the other severity scales.

Results Preliminary results show little differences in the predictive performance of the different severity scales. The accuracy of the model improves significantly when age is added as a predictor.

Conclusions Conclusions will be drawn on the final results. 